\title{
Synovial macrophage populations linked to $\mathrm{RA}$ remission
}

\section{a low ratio \\ $(\leq 2.5)$ of \\ MerTK ${ }^{\text {pos }}$ to \\ MerTK ${ }^{\text {neg }}$ \\ STMs was \\ predictive of \\ disease flare \\ upon drug \\ withdrawal} present during inflammation and
might have tissue homeostatic and regulatory functions," reports Mariola Kurowska-Stolarska, co-corresponding author on the new study. "Thus, we sought to understand the functions of human synovial tissue macrophages (STMs) that are present in healthy joints, and in particular in those patients with RA who achieved and maintained disease remission after treatment discontinuation."

Using single cell RNA sequencing (scRNA-seq), the researchers characterized the STM populations

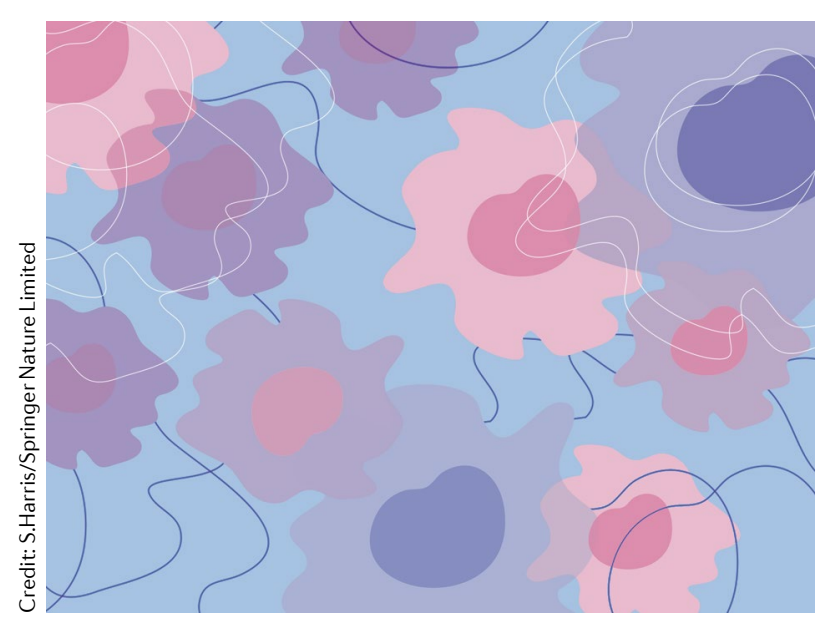

present in the healthy synovium, in the inflamed synovium of patients with active RA and in the synovium of patients with RA in sustained disease remission. They identified two main STM populations: MerTK $^{\text {neg }}$ and MerTK ${ }^{\text {pos }}$ STMs. These two populations contained four and five distinct phenotypic clusters, respectively.

The MerTK ${ }^{\text {neg }}$ STM clusters were expanded in the synovium during active RA, but not during RA in remission. The MerTK ${ }^{\text {neg }}$ population comprised STMs expressing alarmins (CD48 ${ }^{\text {pos }}$ S100A12 $2^{\text {pos }}$ cluster), the bone-remodelling molecule osteopontin (CD48 $8^{\text {pos }} S P P 1^{\text {pos }}$ cluster), an interferon signature (HLA ${ }^{\text {pos ISG15 }}{ }^{\text {pos }}$ cluster) and an antigen-presenting cell signature (HLA ${ }^{\text {pos CLEC10A }}{ }^{\text {pos }}$ cluster).

In vitro analysis found that the MerTK $^{\text {neg }}$ STMs were responsible for the release of pro-inflammatory mediators, including TNF and IL-6, and that these STMs could induce pathological activation of fibroblast-like synoviocytes (FLS) in cocultures.

By contrast, MerTK ${ }^{\text {pos }}$ STMs were predominant in the healthy synovium and during disease remission, in particular in the synovium of patients who maintained remission after drug withdrawal. Notably, a low ratio $(\leq 2.5)$ of MerTK ${ }^{\text {pos }}$ to MerTK ${ }^{\text {neg }}$ STMs was predictive of disease flare upon drug withdrawal.

The MerTK ${ }^{\text {pos }}$ population contained TREM2 ${ }^{\text {pos }}$ and LYVE1 $1^{\text {pos }}$ clusters. These STMs produced little of or no pro-inflammatory mediators in vitro but could produce the pro-resolving lipid mediator resolvin $\mathrm{D} 1$ and also induced repair responses in FLS. "We found that, at least in part, these functions were driven by MerTK and its ligand GAS6." says
Kurowska-Stolarska. "GAS6 was expressed by a specific sublining layer THYpos CXCL14 ${ }^{\text {pos }}$ cluster of FLS, and this expression was increased in FLS of patients in disease remission," continues co-corresponding author Stefano Alivernini.

Transcriptomic analysis identified the presence of a regulatory transcriptomic signature in MerTK ${ }^{\text {pos }}$ STMs from patients with RA in remission that was absent in MerTK ${ }^{\text {pos }}$ STMs from healthy individuals or from patients with active RA. This signature included genes encoding the transcription factors KLF4 and NR4A2.

The findings of this study confirm that not all synovial macrophages are inflammatory and that sustained disease remission is actively maintained by MerTK ${ }^{\text {pos }}$ macrophages.

"The immediate implication of these findings, if validated with additional cohorts of patients with $\mathrm{RA}$ in remission, is an application of the ratio of MerTK ${ }^{\text {pos }}$ to MerTK ${ }^{\text {neg }}$ STMs as a predictor of disease flare versus sustained remission to help with treatment decisions in clinical practise," explains Alivernini. "Our study also provides potential cellular and molecular mechanisms involved in re-instating synovial homeostasis (for example, MerTK-GAS6 or KLF2KLF4 pathways) that, if therapeutically enhanced, could bring more patients to sustained disease remission."

"This project is a brilliant example of the application of scRNA-seq to an important clinical question" adds co-corresponding author and bioinformatician Thomas Otto.

Jessica McHugh

ORIGINAL ARTICLE Alivernini, S. et al. Distinct synovial tissue macrophage subsets regulate inflammation and remission in rheumatoid arthritis. Nat. Med. https://doi.org/10.1038/ s41591-020-0939-8 (2020) 